\title{
Téoros
}

Revue de recherche en tourisme

\section{Un défi particulier : mieux gérer le tourisme en améliorant la formation et les conditions de travail}

\section{Gilbert Tarrab}

Volume 2, numéro 1, février 1983

Formation et conditions de travail en tourisme

URI : https://id.erudit.org/iderudit/1080836ar

DOI : https://doi.org/10.7202/1080836ar

Aller au sommaire du numéro

Éditeur(s)

Université du Québec à Montréal

ISSN

0712-8657 (imprimé)

1923-2705 (numérique)

Découvrir la revue

Citer ce document

Tarrab, G. (1983). Un défi particulier : mieux gérer le tourisme en améliorant la formation et les conditions de travail. Téoros, 2(1), 1-1.

https://doi.org/10.7202/1080836ar d'utilisation que vous pouvez consulter en ligne. 


\author{
Un défi particulier: \\ mieux gérer le tourisme \\ en améliorant la formation \\ et les conditions de travail
}

Quand je fus approché pour préparer ce numéro thématique de la revue Téoros sur "les conditions de travail en tourisme" je ne fus pas long a accepter. Non pas qu'il s'agisse là de ma spécialité, loin de là. Mais il m'a semblé que ce secteur d'activité, essentiel à la fin du XXe siecle, méritait grandement qu'on s'y attarde et qu'on lui consacre le temps qu'il fallait pour preparer un tel numero.

Gérer le tourisme est devenu, en effet, une activité en soi, bien différenciée des autres secteurs de gestion. Non pas tant dans les principes d'une gestion saine, qui sont similaires dans n'importe quel secteur économique et culturel, qu'il soit public ou privé. Mais gérer la "chose" touristique comporte un defi particulier, qu'on ne retrouve pas nécessairement dans les autres secteurs d'activité économique: le capital humain, comme le montre le texte de Claude Jou. hannet, y est peut-etre encore plus essentiel qu'ailleurs, étant donné la nature même du secteur "tourisme". Cela nécessite une formation d'un type particulier, comme s'emploie à le démontrer /article d'André Bergeron, professeur au Cegep de Granby. Multidisciplinaire par essence, cette formation se doit de recouvrir plusieurs champs d'expertise. Dans cet article, André Bergeron et ses collaborateurs sinterrogent notamment sur /adequation entre la formation et les emplois.

Le texte de Michel Grant, aidé dans sa démarche par deux eitudiants en tourisme, fait le point sur les conditions de travail en tourisme, plus particulièrement dans les secteurs de I'hótellerie et de la restauration. Le dernier rapport complet et sérieux sur l'ensemble de l'industrie touristique quebécoise remonte a 1970: il s'agit du fameux rapport Baumann'". Certes, il y eut depuis divers rapports sectoriels mais, à notre connaissance, aucun n'a traité de l'ensemble de la question. Le texte de Michel Grant, sans combler cette lacune, =n attend toujours avec impatience un rapport exhaustif sur l'état de l'industrie touristique dans son ensemble pour les années 1980-, souligne néanmoins plus spécifiquement limportante question de la détermination des conditions de travail et tout ce qui entoure la négociation multipatronale. ${ }^{2 /}$.
Jean Stafford, quant à lui, nous trace un tableau de ce que pourrait être le travail en tourisme en Ian 2000 . Nous avons en effet pensé qu'un texte sur les prospectives et perspectives d'avenir sur le travail en tourisme pourrait, de par les questions et interrogations soulevées, susciter une riche reflexion, voire un debat, sur l'a-venir de la chose touristique au Québec.

Enfin, une bibliographie sélective, realisée par les documentalistes du Centre d'études en tourisme, contient la mention de plusieurs ouvrages, rapports, articles de revue qui révè. lent que les problemes d'emplois que nous avons ici mème au Québec se retrouvent parfois tels quels, dans d'autres nations, beaucoup plus "vieilles" du point de vue historique. Louis Jolin met en parallele quelques extraits de ces ouvrages, ce qui a pour effet d'elargir le debat et les perspectives.

Nous disions au début de cette présentation que gérer le tourisme n'était pas une gestion comme les autres. Gageons que ce numéro aura réussi a montrer quels paramètres or ginaux sont en jeu quand on se donne pour objet et comme discipline de gérer le tourisme. Les variables en sont telle. ment diversifiées que, de plus en p/us, le statut de "science" particulière est en voie de s'actualiser pour sérieusement traiter de cette question. Le temps de ramateurisme et du melting-pot est definitivement révolu. Dorénavant, ce champ d'investigation demandera, tres bientót, des spécialistes formés à l'exercice de cette science.

Gilbert Tarrab

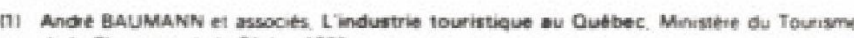
de L Chuse et of is Peche. $19 \%$

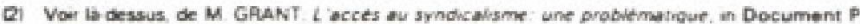
irevue de rassociation des proteswonneis en ressovices humanes du Guebeci november 1919

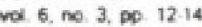

